\title{
IMMERSIONS AND EMBEDDINGS OF TANGENT BUNDLES
}

\author{
FUICHI UCHIDA
}

(Received March 1, 1965)

Introduction. Let $M^{n}$ be an $n$-dimensional compact connected differentiable manifold without boundary. Let $\tau\left(M^{n}\right)=\left(T\left(M^{n}\right), \pi, M^{n}\right)$ be the tangent bundle of $M^{n}$, where $T\left(M^{n}\right)$ is the total space and $\pi: T\left(M^{n}\right) \rightarrow M^{n}$ is the projection, then $T\left(M^{n}\right)$ is a $2 n$-dimensional connected differentiable manifold.

In this paper we consider embeddings and immersions of $T\left(M^{n}\right)$ in Euclidean spaces. For example, in general $M^{n} \subset R^{2 n}$, so $T\left(M^{n}\right) \subset R^{4 n}$, but we get $T\left(M^{n}\right) \subset R^{3 n}$. $M^{n} \subseteq R^{n+k}$ does not always imply $M^{n} \subset R^{n+k}$, but if $k>0$ then for any $T\left(M^{n}\right), T\left(M^{n}\right) \subseteq R^{2 n+k}$ implies $T\left(M^{n}\right) \subset R^{2 n+k}$.

In section 1 , we recall some results of $K O(X)$ and immersion. In sections 2, 3 and 4, we prove some general theorems for immersions and embeddings of $T\left(M^{n}\right)$. And in sections 5 and 6, we consider several applications for the tangent bundles of projective spaces.

Notations $M \subset N$ and $M \subseteq N$ mean " $M$ is differentiably embedded in $N$ " and " $M$ is differentiably immersed in $N$ " respectively. $M \notin N$ and $M \neq N$ means " $M \subset N$ is false" and " $M \subseteq N$ is false" respectively.

1. Known results for $\boldsymbol{K O}(\boldsymbol{X})$. Let $X$ be a finite connected $C W$-complex and $K O_{(k)}(X)$ be the set of isomorphism classes of $k$-dimensional real vector bundles over $X$. Let $\varepsilon(X)$ denote the set of all isomorphism classes of real vector bundles on $X$. Then in our notation

$$
\varepsilon(X)=\bigcup_{k=0}^{\infty} K O_{(k)}(X)
$$

$\varepsilon(X)$ is an abelian semigroup with zero for the Whitney sum. We shall use the symbol $\xi^{k}$ to denote a $k$-dimensional vector bundle and $\varepsilon^{k}$ to denote the trivial bundle of dimension $k$. We have a mapping

$$
i_{k}: K O_{(k)}(X) \rightarrow K O_{(k+1)}(X)
$$

defined by $i_{k}\left(\xi^{k}\right)=\xi^{k} \oplus \varepsilon^{1}$, and denote $\widetilde{K O}(X)$ the direct limit of this sequence and denote $\xi_{0}$ the corresponding class of $\xi^{k}$.

Now we can define the Grothendieck ring of real vector bundles over $X$ 
by setting

$$
K O(X)=\widetilde{K O}(X)+Z
$$

The operations of addition and multiplication in $K O(X)$ are induced by Whitney sum and tensor product of vector bundles. And we have a homomorphism

$$
\theta: \varepsilon(X) \rightarrow K O(X)
$$

defined by $\theta\left(\xi^{k}\right)=\xi_{0}+k$. $\xi_{0}$ in $\widetilde{K O}(X)$ is called the stable class of $\xi^{k}$. Then by the stability of classifying spaces of the orthogonal groups and the definition of $\theta$ we have the following lemma (cf. [6]).

LEMMA 1. (a) $\theta\left(\xi^{k}\right)=\theta\left(\eta^{k}\right)$ if and only if, for some $n, \xi^{k} \oplus \varepsilon^{n}=\eta^{k} \oplus \varepsilon^{n}$. (b) If $\xi^{\prime}\left(\xi^{k}\right)=\theta\left(\eta^{k}\right)$ and $k>\operatorname{dim} X$, then $\xi^{k}=\eta^{k}$.

An element of $K O(X)$ is said to be positive if it is in the image of $\theta$. If $\xi_{0} \in \widetilde{K O}(X)$, the geometrical dimension of $\xi_{0}$, written $g\left(\xi_{0}\right)$, is the least integer $k$ such that $\xi_{0}+k$ is positive.

Let $f: X \rightarrow Y$ be a continuous map. Then we denote $f^{\prime}\left(\xi^{k}\right)$ the induced bundle of $\xi^{k}$. Then we get the following properties of the function $g$ (cf. [6]).

LEMMA 2. (a) If $\xi_{0} \in \widetilde{K O}(X)$, then $g\left(\xi_{0}\right) \leqq \operatorname{dim} X$. (b) If $\xi_{0}, \eta_{0} \in \widetilde{K O}(X)$, then $g\left(\xi_{0}+\eta_{0}\right) \leqq g\left(\xi_{0}\right)+g\left(\eta_{0}\right)$. (c) If $f: X \rightarrow Y$ is a map and $\xi_{0} \in \widetilde{K O}(Y)$, then $g\left(\xi_{0}\right) \geqq g\left(f !\left(\xi_{0}\right)\right)$.

Now $M^{n}$ will denote a connected $n$-dimensional differentiable manifold and $\tau^{n}$ will denote its tangent bundle. Using the geometrical dimension, the main theorem of Hirsch on immersion of manifolds in Euclidean spaces becomes ([2], [6]),

THEOREM 3. For $k>0, M^{n} \subseteq R^{n+k}$ if and only if $g\left(-\tau_{0}\right) \leqq k$.

2. Tangent bundle of $\boldsymbol{T}\left(\boldsymbol{M}^{n}\right)$. Let $M^{n}$ be an $n$-dimensional compact connected differentiable manifold without boundary. Let $T\left(M^{n}\right)$ be the total space of the tangent bundle $\tau\left(M^{n}\right)$ of $M^{n}$.

Let us now consider the projection $\pi: T\left(M^{n}\right) \rightarrow M^{n}$ and the inclusion $i: M^{n} \rightarrow T\left(M^{n}\right)$, then we have

LEMMA 4. (a) $i^{!}\left(\tau\left(T\left(M^{n}\right)\right)\right)=\tau\left(M^{n}\right) \oplus \tau\left(M^{n}\right)$. 
(b) $\tau\left(T\left(M^{n}\right)\right)=\pi^{!}\left(\tau\left(M^{n}\right) \oplus \tau\left(M^{n}\right)\right)$.

Proof. Let $\left\{U_{\alpha}\right\}$ be a local chart of $M^{n}$ and as usual $\left\{\pi^{-1}\left(U_{\alpha}\right)\right\}$ be a local chart of $T\left(M^{n}\right)$. Let $U_{\alpha} \cap U_{\beta} \neq \varnothing$ and $\left(x^{1}, \cdots, x^{n}\right),\left(\bar{x}^{1}, \cdots, \bar{x}^{n}\right)$ be local coordinates for $U_{\alpha}, U_{\beta}$ respectively and $\left(x^{1}, \cdots, x^{n}, a^{1}, \cdots, a^{n}\right)$, $\left(\bar{x}^{1}, \cdots, \bar{x}^{n}, \bar{a}^{1}, \cdots, \bar{a}^{n}\right)$ be local coordinates for $\pi^{-1}\left(U_{\alpha}\right), \pi^{-1}\left(U_{\beta}\right)$ respectively. Consider the tangent vectors on $U_{\alpha} \cap U_{\beta}$, then

$$
\sum_{i} a^{i} \frac{\partial}{\partial x^{i}}=\sum_{i} \bar{a}^{i} \frac{\partial}{\partial \bar{x}^{i}}
$$

thus

$$
\bar{a}^{i}=\sum_{k} a^{k} \frac{\partial x^{i}}{\partial x^{k}}
$$

Therefore the coordinate transformation of $\tau\left(M^{n}\right)$ is given by

$$
g_{\beta \alpha}(p)=\left(\frac{\partial \bar{x}^{i}}{\partial x^{j}}(p)\right), \quad p \in U_{\alpha} \cap U_{\beta}
$$

Similarly the coordinate transformation of $\tau\left(T\left(M^{n}\right)\right)$ is given by

$$
\bar{g}_{\beta a}(q)=\left(\begin{array}{ll}
\frac{\partial \bar{x}^{i}}{\partial x^{j}} & \frac{\partial \bar{x}^{i}}{\partial a^{j}} \\
\frac{\partial \bar{a}^{i}}{\partial x^{j}} & \frac{\partial \bar{a}^{i}}{\partial a^{j}}
\end{array}\right), \quad q \in \pi^{-1}\left(U_{\alpha}\right) \cap \pi^{-1}\left(U_{\beta}\right)
$$

here

$$
\frac{\partial \bar{x}^{i}}{\partial a^{j}}=0, \frac{\partial \bar{a}^{i}}{\partial x^{j}}=\sum_{k} a^{k} \frac{\partial^{2} \bar{x}^{i}}{\partial x^{j} \partial x^{k}}, \frac{\partial \bar{x}^{i}}{\partial a^{j}}=\sum_{k} \frac{\partial a^{k}}{\partial a^{j}} \frac{\partial \bar{x}^{i}}{\partial x^{k}}=\frac{\partial \bar{x}^{i}}{\partial x^{j}} .
$$

If $q=i(p), p \in U_{a} \cap U_{\beta}$ then $a^{1}=\cdots=a^{n}=0$, consequently

$$
\bar{g}_{\beta \alpha}(q)=\left(\begin{array}{cc}
\frac{\partial \bar{x}^{i}}{\partial x^{j}}(p) & 0 \\
0 & \frac{\partial \bar{x}^{i}}{\partial x^{j}}(p)
\end{array}\right)
$$


This means $i^{!}\left(\tau\left(T\left(M^{n}\right)\right)\right)=\tau\left(M^{n}\right) \oplus \tau\left(M^{n}\right)$. Since $i \circ \pi$ is a strong deformation retraction, we have $\tau\left(T\left(M^{n}\right)\right)=\pi^{!}\left(\tau\left(M^{n}\right) \oplus \tau\left(M^{n}\right)\right)$.

'THeOREM 5. For $k>0, T\left(M^{n}\right) \subseteq R^{2 n+k}$ implies $T\left(M^{n}\right) \subset R^{2 n+k}$.

PROOF. Consider an immersion $f: T\left(M^{n}\right) \rightarrow R^{2 n+k}$, then

$$
\tau\left(T\left(M^{n}\right)\right) \oplus \nu_{f}=\varepsilon^{2 n+k},
$$

where $\nu_{f}$ is the normal bundle of the immersion $f$. By Lemma 4,

$$
\boldsymbol{\tau}\left(M^{n}\right) \oplus \boldsymbol{\tau}\left(M^{n}\right) \oplus i^{!} \nu_{f}=\varepsilon^{2 n+k} .
$$

Let now $h: M^{n} \rightarrow R^{2 n+k}$ be an embedding, then

$$
\tau\left(M^{n}\right) \oplus \nu_{h}=\varepsilon^{2 n+k},
$$

where $\nu_{h}$ is the normal bundle of the embedding $h$. Hence we have

$$
\theta\left(\tau\left(M^{n}\right) \oplus i ! \nu_{f}\right)=\theta\left(\nu_{h}\right)
$$

By Lemma 1(b) and $k>0$, we get

$$
\boldsymbol{\tau}\left(M^{n}\right) \oplus i ! \nu_{f}=\nu_{h} .
$$

Therefore we can embed $\tau\left(M^{n}\right)$ as a subbundle of $\nu_{h}$. And if we consider a tubular neighborhood for the embedding $h$, we have $T\left(M^{n}\right) \subset R^{2 n+k}$.

\section{Immersion and geometrical dimension.}

THeOREM 6. For $k>0, T\left(M^{n}\right) \subseteq R^{2 n+k}$ if and only if $g\left(-2 \tau_{0}\left(M^{n}\right)\right) \leqq k$.

ProOF. By Lemma 4, $g\left(-\tau_{0}\left(T\left(M^{n}\right)\right)\right)=g\left(-2 \tau_{0}\left(M^{n}\right)\right)$. And the result follows from Theorem 3 .

COROllaRy 7. $T\left(M^{n}\right) \subset R^{3 n}$.

PROOF. Since $g\left(-2 \tau_{0}\left(M^{n}\right)\right) \leqq n$, by Lemma 2 (a), the result follows from Theorem 5 and 6 .

Let now $\pi: E \rightarrow B$ be a differentiable principal fibre bundle, then (cf. [4], Ch. II, §1) 


$$
\tau(E)=\pi^{!}(\tau(B)) \oplus \varepsilon^{m}, m=\operatorname{dim} E-\operatorname{dim} B .
$$

Therefore

$$
\tau(T(E))=\bar{\pi}^{!}(\tau(T(B))) \oplus \varepsilon^{2 m},
$$

where $\bar{\pi}: T(E) \rightarrow T(B)$ is induced by $\pi$. Following Lemma 2 (c) and Theorem 3 , we have

THEOREM 8. Let $\pi: E \rightarrow B$ be a differentiable principal fibre bundle, then $T(B) \subseteq R^{2 \mathrm{dim} B+k}$ implies $T(E) \subseteq R^{2 \mathrm{dim} E+k}$ for $k>0$.

Let $\lambda^{i}$ be the exterior $i$-power operation in $\varepsilon(X)$. Atiyah ([1]) modifies $\lambda^{i}$ and introduces an operation $\gamma^{i}$ in $K O(X)$, and a homomorphism

$$
\gamma_{t}(\xi)=\sum_{i=0}^{\infty} \gamma^{i}(\xi) t^{i}
$$

of $K O(X)$ to the multiplicative group $A(X)$ of formal power series in $t$ with coefficients in $K O(X)$ and constant term 1 . And he has

PROPOSITION 9. If $\xi_{0} \in \widetilde{K O}(X)$, then $\gamma^{i}\left(\xi_{0}\right)=0$ for $i>g\left(\xi_{0}\right)$.

PROPOSITION 10. Let $M^{n}$ be a compact differentiable manifold of dimension $n$. If $M^{n} \subset R^{n+k}$, then $\gamma^{i}\left(-\tau_{0}\right)=0$ for $i \geqq k$.

Let now $M^{n}$ be an $n$-dimensional compact differentiable manifold without boundary. By simple modification of the proof of Proposition 10, we have

THEOREM 11. For $k>0, T\left(M^{n}\right) \subset R^{2 n+k}$ implies $\gamma^{i}\left(-2 \tau_{0}\right)=0$ for $i \geqq k$.

4. Immersion and Stiefel Whitney class. The projection $\pi: T\left(M^{n}\right) \rightarrow M^{n}$ induces an onto isomorphism $\pi^{*}: H^{*}\left(M^{n}, Z_{2}\right) \rightarrow H^{*}\left(T\left(M^{n}\right), Z_{2}\right)$. Using Lemma 4 , we have a relation

$$
\bar{w}\left(T\left(M^{n}\right)\right)=\pi^{*}\left(\bar{w}\left(M^{n}\right) \cdot \bar{w}\left(M^{n}\right)\right)
$$

between the dual Stiefel Whitney classes for $M^{n}$ and $T\left(M^{n}\right)$. Denote $\vec{w}_{i}\left(M^{n}\right)$. the $i$-dimensional dual Stiefel Whitney class of $M^{n}$.

THEOREM 12. $\sum_{i=0}^{k} \bar{w}_{i}\left(M^{n}\right) \cdot \bar{w}_{k-i}\left(M^{n}\right) \neq 0$ implies $T\left(M^{n}\right) \neq R^{2 n+k-1}$. 
Proof. This condition is equivalent to $\bar{w}_{k}\left(T\left(M^{n}\right)\right) \neq 0$ and the result is obvious (cf. [3]).

Now consider the normal bundle $\nu^{n}$ of an embedding $T\left(M^{n}\right) \subset R^{3 n}$. Then $T\left(M^{n}\right) \subseteq R^{3 n-k}$ if and only if the bundle associated to $\nu^{n} \oplus \varepsilon^{m}$ with fibre $V_{n+m, k+m}$ has a cross-section for some $m \geqq 0$, because the existence of such a cross-section is equivalent to $g\left(\nu_{0}\right) \leqq n-k$. Since $H^{i}\left(T\left(M^{n}\right), G\right)=0$ for $i>n$ and for any local coefficient $G$, we get the following results.

THEOREM 13. (a) Suppose $n$ is even, then $T\left(M^{n}\right) \subseteq R^{3 n-1}$ if and only if $\sum_{i=0}^{n} \bar{w}_{i}\left(M^{n}\right) \cdot \bar{w}_{n-i}\left(M^{n}\right)=0$. (b) Suppose $n=4 k+1$, then $T\left(M^{n}\right) \subseteq R^{3 n-2}$ if and only if $\sum_{i=0}^{n-1} \bar{w}_{i}\left(M^{n}\right) \cdot \bar{w}_{n-i-1}\left(M^{n}\right)=0$.

PROOF. The conditions are the first obstructions to the existence of the desired cross-sections. For (b), the second obstruction has values in

$$
\pi_{4 k}\left(V_{n+1,3}\right)=0 \quad([5]) .
$$

5. The tangent bundles of projective spaces. $F P_{n}$ denotes projective space of dimension $n$ over the field $F$, where $F$ is $R, C$ or $H$, the real, complex, or quaternion number field. Then $F P_{n}$ is a compact connected differentiable manifold of $d n$ dimensions, where $d$ is the dimension of $F$ over $R$.

Let $\alpha_{F} \in H^{d}\left(F P_{n}, Z_{2}\right)$ be the generator of the cohomology algebra $H^{*}\left(F P_{n}, Z_{2}\right)$. Then the total Stiefel Whitney class of $F P_{n}$ is

$$
w\left(F P_{n}\right)=\left(1+\alpha_{F}\right)^{n+1}
$$

and if $f(n)$ is the integer defined by $2^{f(n)-1} \leqq n<2^{f(n)}$, the dual Stiefel Whitney class of $F P_{n}$ is

$$
\bar{w}\left(F P_{n}\right)=\left(1+\alpha_{F}\right)^{2^{F(n)}-n-1}
$$

Therefore, by (4.1)

$$
\pi^{*-1}\left(\bar{w}\left(T\left(F P_{n}\right)\right)\right)=\left(1+\alpha_{F}\right)^{2\left(2^{f(n)}-n-1\right)}
$$

Using Theorems 6,12, Corollary 7 and (5.2), we have the following table for $T\left(R P_{n}\right) \subset R^{m}$ and $T\left(R P_{n}\right) \neq R^{k}$. 


\begin{tabular}{|c|rrrrrrrr}
\hline$n$ & 1 & 2 & 3 & 4 & 5 & 6 & 7 & 8 \\
\hline$m$ & 2 & 6 & 7 & 12 & 14 & 14 & 15 & 24 \\
$k$ & 1 & 5 & $*$ & 11 & 13 & 13 & $*$ & 23 \\
\hline
\end{tabular}

REMARK. If $M^{n}$ is an $n$-dimensional compact differentiable manifold without boundary, we have $M^{n} \neq R^{n}$. But $T\left(M^{n}\right)$ is not compact, so I cannot know whether, for $n=3$ or $7, T\left(R P_{n}\right)$ can be immersed in $R^{2 n}$.

Let $\pi_{1}: R P_{2 n+1} \rightarrow C P_{n}$ and $\pi_{3}: R P_{4 n+3} \rightarrow H P_{n}$ be the principal fibre bundles considered in [6]. Then by Theorem 8 ,

THEOREM 14. For $k>0$, (a) $T\left(C P_{n}\right) \subseteq R^{4 n+k}$ implies $T\left(R P_{2 n+1}\right) \subseteq R^{1 n+2+k}$. (b) $T\left(H P_{n}\right) \subseteq R^{8 n+k}$ implies $T\left(R P_{4 n+3}\right) \subseteq R^{8 n+6+k}$.

COROLlaRY 15. (a) $T\left(R P_{n}\right) \subseteq R^{3 n-1}$ for $n=2 s+1$. (b) $T\left(R P_{n}\right) \subseteq R^{3 n-3}$ for $n=4 s+3$ and $s>0$.

In order to calculate the binomial coefficients $\bmod p$, the following lemma is useful. The proof is easy.

LEMMA. Let $p$ be a prime and let $a=\sum_{i=0}^{m} a_{i} p^{i}$ and $b=\sum_{i=0}^{m} b_{i} p^{i}\left(0 \leqq a_{i}\right.$, $\left.b_{i}<p\right)$. Then

$$
\left(\begin{array}{l}
b \\
a
\end{array}\right) \equiv \prod_{i=0}^{m}\left(\begin{array}{l}
b_{i} \\
a_{i}
\end{array}\right) \bmod p
$$

By (4.1) and (5.1), di-dimensional dual Stiefel Whitney class $\bar{w}_{d i}\left(T\left(F P_{n}\right)\right)$ $=0$ if and only if

$$
\left(\begin{array}{c}
2\left(2^{f(n)}-n-1\right) \\
i
\end{array}\right) \equiv \equiv \bmod 2
$$

For example, we have

THEOREM 16. Let $d$ be the dimension of $F$ over $R$. Then $T\left(F P_{n}\right)$ ₹ $R^{3 d n-1}$ for $n=\sum_{i=1}^{r} a_{i} 2^{i s-t}\left(a_{i}=0\right.$ or $\left.1, s>1,0 \leqq t<s\right)$. 
THEOREM 17. $T\left(R P_{n}\right) \subseteq R^{3 n-4}$ for $n=8 s+7$ and $s>0$.

PROOF. If $k$ is odd, then $\bar{w}_{4 k}\left(T\left(H P_{k}\right)\right)=0$ by (5.3). And following Theorem 13 (a), $T\left(H P_{k}\right) \subseteq R^{12 k-1}$. The result follows from Theorem 14 (b).

From these results, we have many informations on the best possible immersions and embeddings of the tangent bundles of projective spaces in Euclidean spaces.

6. Application of the operation $\gamma^{i}$. We shall now apply Theorem 11 to $R P_{n}$, the $n$-dimensional real projective space. Let $\eta_{0} \in \widetilde{K O}\left(R P_{n}\right)$ denote the stable class of the Hopf bundle $\eta$ on $R P_{n}$. Then $\widetilde{K O}\left(R P_{n}\right)$ is a cyclic group of order $2^{\phi(n)}$ with generator $\eta_{0}$, where $\phi(n)$ is defined as the number of integers $s$ with

$$
0<s \leqq n \text { and } s \equiv 0,1,2 \text { or } 4 \bmod 8 \text {. }
$$

Since $\eta^{2}=1,\left(\eta_{0}\right)^{2}=-2 \eta_{0}$. It is well known that the tangent bundle $\tau=\tau\left(R P_{n}\right)$ is given by

$$
\tau=(n+1) \eta-1 \quad \text { in } K O\left(R P_{n}\right)
$$

and so

$$
\tau_{0}=(n+1) \eta_{0}
$$

Hence

$$
\gamma_{t}\left(-2 \tau_{0}\right)=\left(1+\eta_{0} t\right)^{-2(n+1)}
$$

and so

$$
\gamma^{i}\left(-2 \tau_{0}\right)= \pm\left(\begin{array}{c}
2 n+1+i \\
i
\end{array}\right) \eta_{0}{ }^{i}= \pm 2^{i-1}\left(\begin{array}{c}
2 n+1+i \\
i
\end{array}\right) \eta_{0}
$$

Hence $\gamma^{i}\left(-2 \tau_{0}\right)=0$ if and only if

$$
2^{i-1}\left(\begin{array}{c}
2 n+1+i \\
i
\end{array}\right) \equiv 0 \bmod 2^{\phi(n)}
$$

Let us now define $\rho(n)$ to be the largest integer $s$ for which $2^{s-1}\left(\begin{array}{c}2 n+1+s \\ s\end{array}\right)$ 
is not divisible by $2^{\phi(n)}$. Then Theorem 11 gives

THEOREM 18. $T\left(R P_{n}\right) \phi R^{3 n+\rho(n)}$, if $\rho(n)>0$.

Table for $\boldsymbol{T}\left(\boldsymbol{R} \boldsymbol{P}_{n}\right) \subset \boldsymbol{R}^{m}$ and $\boldsymbol{T}\left(\boldsymbol{R} \boldsymbol{P}_{n}\right) \neq \boldsymbol{R}^{k}$. In the following, * means " $m$ is the best possible immersion" and the parenthesized numbers are given by Theorem 18 .

\begin{tabular}{|r|rrrrrrrrrrrrr|}
\hline$n$ & 9 & 10 & 11 & 12 & 13 & 14 & 15 & 16 & 17 & 18 & 19 & 20 & 21 \\
\hline$m$ & 26 & 30 & 30 & 35 & 37 & 41 & 41 & 48 & 50 & 54 & 54 & 60 & 62 \\
$k$ & $*$ & $*$ & $*$ & $(30)$ & $(30)$ & $(32)$ & $(34)$ & $*$ & $*$ & $*$ & $*$ & $*$ & $*$ \\
\hline
\end{tabular}

\begin{tabular}{|c|ccccccccccccc|}
\hline$n$ & 22 & 23 & 24 & 25 & 26 & 27 & 28 & 29 & 30 & 31 & 32 & 33 & 34 \\
\hline$m$ & 65 & 65 & 71 & 73 & 77 & 77 & 83 & 85 & 89 & 89 & 96 & 98 & 102 \\
$k$ & 61 & 61 & 61 & $(62)$ & $(62)$ & $(62)$ & $(68)$ & $(70)$ & $(70)$ & $(72)$ & $*$ & $*$ & $*$ \\
\hline
\end{tabular}

\begin{tabular}{|r|rrrrrrrrrrrr|}
\hline$n$ & 35 & 36 & 37 & 38 & 39 & 40 & 41 & 42 & 43 & 44 & 45 & $\cdots$ \\
\hline$m$ & 102 & 108 & 110 & 113 & 113 & 120 & 122 & 126 & 126 & 131 & 133 & \\
$k$ & $*$ & $*$ & $*$ & 109 & 109 & $*$ & $*$ & $*$ & $*$ & 125 & 125 & \\
\hline
\end{tabular}

\begin{tabular}{|r|rrrrrrrrrrrrr|}
\hline$n$ & 63 & 64 & 65 & 66 & 67 & 68 & 69 & 70 & 71 & 72 & 73 & 74 & 75 \\
\hline$m$ & 185 & 192 & 194 & 198 & 198 & 204 & 206 & 209 & 209 & 216 & 218 & 222 & 222 \\
$k$ & $(150)$ & $*$ & $*$ & $*$ & $*$ & $*$ & $*$ & 205 & 205 & $*$ & $*$ & $*$ & $*$ \\
\hline
\end{tabular}

\begin{tabular}{|l|rrrrrrrrrrrr|}
\hline$n$ & 76 & 77 & 78 & 79 & 80 & 81 & 82 & 83 & 84 & 85 & 86 & $\cdots$ \\
\hline$m$ & 227 & 230 & 233 & 233 & 240 & 242 & 246 & 246 & 252 & 254 & 257 & \\
$k$ & 221 & 221 & 221 & 221 & $*$ & $*$ & $*$ & $*$ & $*$ & $*$ & 253 & \\
\hline
\end{tabular}


REMARK. $T\left(H P_{6}\right) \subset R^{11}$ by means of Theorem 13 (a), so we have $T\left(R P_{27}\right) \subset R^{77}$ by Theorem $14(\mathrm{~b})$.

ADDED IN PROOF. If $M^{n}$ is an orientable $\pi$-manifold. then $M^{n}$ is immersible in $R^{n+1}$ so that its normal bundle is trivial. This shows $M^{n} \times R^{1}$ $\subseteq R^{n+1}$. Consequently, if $M^{n}$ is parallelizable, then $T\left(M^{n}\right) \subseteq R^{2 n}$. For example, $T\left(R P_{3}\right) \subseteq R^{6}$ and $T\left(R P_{7}\right) \subseteq R^{14}$.

\section{REFERENCES}

[1] M. F. ATтYAH, Immersions and embeddings of manifolds, Topology, 1(1962), 125-132.

[2] M. W. Hirsch, Immersions of manifolds, Trans. Amer. Math. Soc., 93(1959), 242276.

[3] J. MilnoR, Lectures on characteristic classes, Princeton, (1957).

[4] K. NomizU, Lie groups and differential geometry, The Mathematical Society of Japan, (1956).

[5] G. F. PAechteR, On the groups $\pi_{r}\left(V_{n, m}\right), 1$, Quart. Journ. Math. (Oxford), 7(1956), 249-258.

[6] B. J. SANDERSON, Immersions and embeddings of projective spaces, Proc. London Math. Soc., 14(1964), 137-153.

[7] N. E. STEEnROD, The topology of fibre bundles, Princeton Univ. (1951).

TÔHOKU UNIVERSITY. 